\title{
Using Differentials to Bridge the Vector Calculus Gap
}

Tevian Dray and Corinne A. Manogue
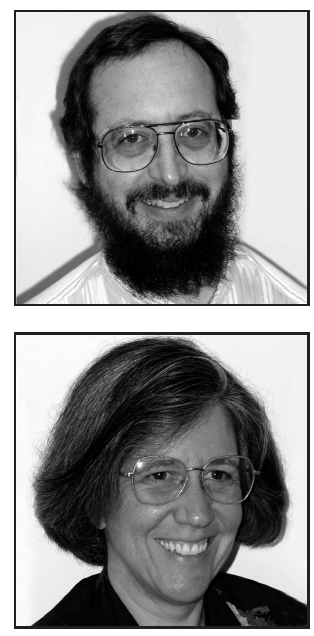

Tevian Dray (tevian@math.orst.edu) received his B.S. from MIT in 1976, his Ph.D. from Berkeley in 1981, spent several years as a physics postdoc, and is now Professor of Mathematics at Oregon State University. He considers himself a mathematician, but isn't sure. (Neither is his department.) Most of his research has involved general relativity.

Corinne Manogue (corinne@physics.orst.edu) received her A.B. from Mount Holyoke College in 1977, her Ph.D. from the University of Texas in 1984, and is now Professor of Physics at Oregon State University. She continues to be amazed to find herself a physicist. Most of her research has been related to quantum gravity and superstring theory.

Corinne and Tevian have collaborated on many projects, including two children. In addition to their work on curriculum reform, they are trying to give a unified description of the fundamental particles of nature in terms of the octonions. In 2001-2002 they were the Hutchcroft Visiting Professors of Mathematics at Mount Holyoke College.

\section{Introduction}

There is a surprisingly large gap between the way mathematicians on the one hand and physical scientists and engineers on the other do vector calculus [1]. This gap is particularly apparent when students attempt to apply the vector calculus they have learned in mathematics courses to the problems in electromagnetism that they encounter in subsequent physics or engineering courses. In an effort to bridge this gap, we propose a geometric approach using differentials to some problems in vector calculus.

Our focus is on line and surface integrals. We will stereotype the two traditional points of view as "mathematics" and "physics"; this reflects our own training, but should not be construed as a definitive characterization. It is instructive to contrast the treatment of this material in mathematics and physics textbooks. An excellent presentation of the traditional mathematics approach can be found in Stewart [2], while the physics approach is beautifully described in Griffiths [3]. Our work has been strongly influenced by previous efforts to bridge the gap, including the classic description by Schey [4] of the physics view of divergence and curl, some of which (and more) has been thoughtfully incorporated in the more recent multivariable calculus text by the Calculus Consortium [5]. However, our use of differentials does not appear in any of these books.

How do mathematicians do surface integrals? First, one needs a vector parameterization $\overrightarrow{\boldsymbol{r}}(u, v)$ of the surface. Next, one computes the normal vector

$$
\vec{N}=\frac{\partial \overrightarrow{\boldsymbol{r}}}{\partial u} \times \frac{\partial \overrightarrow{\boldsymbol{r}}}{\partial v}
$$


One can now either compute the scalar surface element $d S=|\vec{N}| d u d v$, or the vector surface element $d \overrightarrow{\boldsymbol{S}}=\hat{\boldsymbol{n}} d S=\overrightarrow{\boldsymbol{N}} d u d v$. Finally, one inserts the surface element into the given surface integral, determines the limits on $u$ and $v$, and evaluates the resulting double integral.

How do physicists do surface integrals? A typical such integral would be the flux of a vector field $\overrightarrow{\boldsymbol{F}}$ through a sphere. The scalar surface element on a sphere is easy to deduce from an infinitesimal rectangle, and the unit normal vector is just $\hat{\boldsymbol{r}}$, the unit vector in the radial direction. Determine $\overrightarrow{\boldsymbol{F}} \cdot \hat{\boldsymbol{n}}$ and integrate over the sphere; the limits are obvious.

Is there a way to combine both perspectives? We think so.

We use a geometric approach that combines the generality of the "mathematics" approach with the ability to handle easily the highly symmetric surfaces typical of the "physics" approach. It also forces students to develop geometric reasoning skills; vector calculus is nearly the only subject remaining in lower-division mathematics where this can be achieved!

Geometric reasoning is one of the fundamental skills of mathematics, along with more traditional algebra skills and increasingly successful numerical techniques. Students in the physical sciences and engineering, who make up a significant fraction of the audience in second-year calculus courses, will rely heavily on geometric reasoning in their careers. But all students benefit from the interplay between different viewpoints.

Some readers may feel that getting students to think geometrically imposes an additional burden on an already overcrowded syllabus. We disagree, although we can offer only anecdotal evidence to the contrary. We taught one of two sections of Calculus III in the fall of 2001 at Mount Holyoke College, a liberal arts college strong in the sciences but without an engineering program. The other section was taught more traditionally, although both sections used the same text [5], and roughly the same syllabus. Both classes made a point of covering the entire book, including Stokes' Theorem. On their evaluations, many students in the other section complained about the pace. None of ours did so; indeed, several commented that the pace was about right. This suggests to us that mastering geometric reasoning makes students more comfortable with the material.

We acknowledge that mathematics teachers often have difficulty dealing with differentials, typically getting bogged down in asking just what, precisely, they are. While we will not address this important issue in detail here, the use of differentials can be rigorously justified, either as a shorthand notation for Riemann sums or in terms of differential forms. We wish to emphasize, however, that an approach based on differentials closely reflects the way most scientists and engineers successfully use calculus.

\section{Vector differentials}

Given a curve, it is natural to consider the infinitesimal displacement $d s$ along that curve. It is even more fundamental to consider the infinitesimal vector displacement $d \overrightarrow{\boldsymbol{r}}$ along the curve, whose magnitude is of course $d s$, that is $d s=|d \overrightarrow{\boldsymbol{r}}|$, and whose direction is tangent to the curve (see Figure 1).

In solving problems, one often chooses coordinates, for example rectangular coordinates $(x, y)$ in the plane. For arclength, one then constructs an infinitesimal "triangle"1

\footnotetext{
${ }^{1}$ When dealing with infinitesimals, we prefer to avoid second-order errors by anchoring all quantities to the same point.
} 


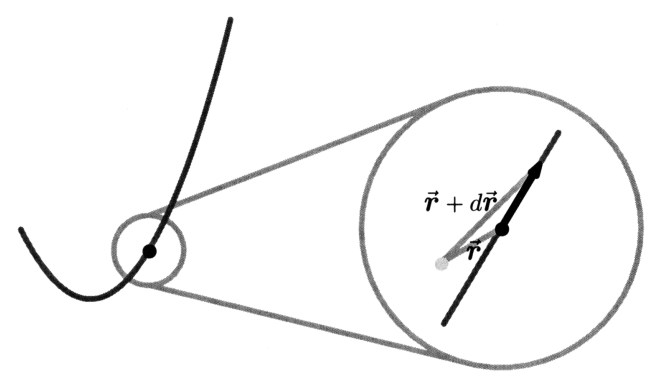

Figure 1. The infinitesimal displacement vector $d \overrightarrow{\boldsymbol{r}}$ along a curve, shown in an "infinite magnifying glass". In this and subsequent figures, artistic license has been taken in the overall scale and the location of the origin in order to make a pedagogical point.

with sides $d x$ and $d y$ and hypotenuse $d s$ in order to arrive at the Pythagorean relation

$$
d s^{2}=d x^{2}+d y^{2}
$$

as shown in the first drawing in Figure 2. But this relationship is really a vector relationship: the sides are the infinitesimal vectors $d x \hat{\boldsymbol{\imath}}$ and $d y \hat{\boldsymbol{j}}$, and the hypotenuse is the infinitesimal displacement $d \overrightarrow{\boldsymbol{r}}$ along the curve, so that

$$
d \overrightarrow{\boldsymbol{r}}=d x \hat{\boldsymbol{\imath}}+d y \hat{\boldsymbol{J}}
$$

as shown in the second of Figure 2. The Pythagorean relationship (1) follows from (2) by taking the (squared) magnitude of each side using dot products.

It is important to realize that $d \overrightarrow{\boldsymbol{r}}$ and $d s$ are defined geometrically, not by (2) and (1). To emphasize this coordinate-independent nature of $d \overrightarrow{\boldsymbol{r}}$, it is useful to study $d \overrightarrow{\boldsymbol{r}}$ in another coordinate system, such as polar coordinates $(r, \phi)$ in the plane. ${ }^{2}$ It is also natural to introduce basis vectors $\{\hat{\boldsymbol{r}}, \hat{\boldsymbol{\phi}}\}$ adapted to these coordinates, with $\hat{\boldsymbol{r}}$ being the unit vector in the radial direction, and $\hat{\phi}$ being the unit vector in the direction of increasing $\phi .^{3}$
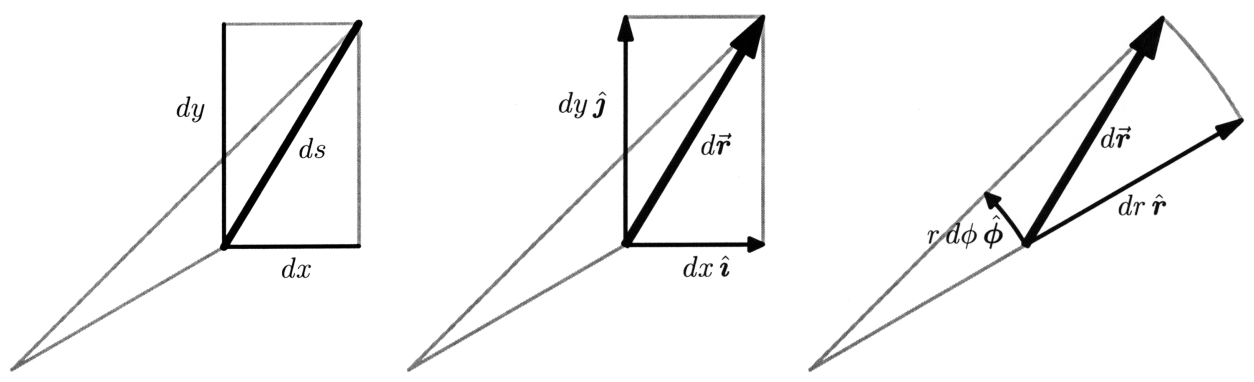

Figure 2. The first figure shows the infinitesimal version of the Pythagorean Theorem, while the remaining two figures show the vector version of the same result, in rectangular and polar coordinates, respectively.

\footnotetext{
${ }^{2}$ We choose $\phi$ for the polar angle in order to agree with the standard conventions for spherical coordinates used by everyone but mathematicians; this is further discussed in [6].

${ }^{3}$ One can of course relate $\hat{\boldsymbol{r}}$ and $\hat{\boldsymbol{\phi}}$ to $\hat{\boldsymbol{\imath}}$ and $\hat{\boldsymbol{j}}$, but in most physical applications (as opposed to problems in calculus textbooks) this can be avoided by making an appropriate initial choice of coordinates.
} 
Determining the lengths of the sides of the resulting infinitesimal polar "triangle", one obtains

$$
d \overrightarrow{\boldsymbol{r}}=d r \hat{\boldsymbol{r}}+r d \phi \hat{\phi}
$$

as shown in the last drawing of Figure 2. Analogous expressions can be derived for $d \overrightarrow{\boldsymbol{r}}$ in other coordinate systems, some of which we will use below.

\section{Line integrals}

Suppose you want to find the work done by the force $\overrightarrow{\boldsymbol{F}}=x \hat{\boldsymbol{\imath}}+y \hat{\boldsymbol{j}}$ when moving along a given curve $C$, using the formula

$$
W=\int_{C} \overrightarrow{\boldsymbol{F}} \cdot d \overrightarrow{\boldsymbol{r}}
$$

Consider the parametric curve $\overrightarrow{\boldsymbol{r}}=\left(1-t^{2}\right) \hat{\boldsymbol{\imath}}-t \hat{\boldsymbol{j}}$ with $t \in[0,1]$. It is straightforward to compute

$$
d \overrightarrow{\boldsymbol{r}}=(-2 t \hat{\boldsymbol{\imath}}-\hat{\boldsymbol{j}}) d t
$$

Since in this case the given vector field $\overrightarrow{\boldsymbol{F}}$ is just $\overrightarrow{\boldsymbol{r}}$ itself, we get

$$
\int_{C} \overrightarrow{\boldsymbol{F}} \cdot d \overrightarrow{\boldsymbol{r}}=\int_{0}^{1}\left(\left(1-t^{2}\right)(-2 t)+t\right) d t=\int_{0}^{1}\left(2 t^{3}-t\right) d t=0 .
$$

In physical applications, one is rarely given an explicit parameterization of a curve, but rather some other description. Perhaps the key problem-solving strategy we teach our students is to use what they know, rather than trying to apply a set strategy to all problems of a given type. In particular, we discourage students from explicitly parameterizing curves unless they have to. This "use what you know" philosophy is especially powerful in the context of vector line integrals.

For instance, the curve just discussed might have been defined by the equation $x=$ $1-y^{2}$. Then $d x=-2 y d y$, and substituting into (2) leads to

$$
d \overrightarrow{\boldsymbol{r}}=(-2 y \hat{\boldsymbol{\imath}}+\hat{\boldsymbol{j}}) d y .
$$

The computation is almost the same as before, using $y$ instead of $t$. Alternatively, one could have solved for $y$, then computed $d y$ in terms of $d x$.

It is empowering for students to learn that all these approaches will work, some more quickly than others. This skill can be nicely emphasized by an appropriate group activity, during which different groups report on different approaches. The point is that using what you know will always yield correct answers-eventually.

In a "use what you know" strategy, students sometimes don't know when to stop. For example, they may correctly substitute for $d y$ in terms of $d x$ in $d \overrightarrow{\boldsymbol{r}}$, but forget to substitute for $y$ in terms of $x$ in $\overrightarrow{\boldsymbol{F}}$. The rule of thumb that students should learn for line integrals is that they shouldn't start integrating until they have the integral in terms of a single parameter, including correctly determining the limits in terms of it. Lines are one-dimensional! 
One final point: vector line integrals of the form $\int \overrightarrow{\boldsymbol{F}} \cdot d \overrightarrow{\boldsymbol{r}}$ are directed integrals (unlike integrals with respect to arclength, of the form $\int f d s$ ); the sign of the answer depends on which way you traverse the curve. Students will obtain the correct sign automatically if they integrate from the beginning point to the final point, without putting in any artificial signs. This may result in an integral that goes from a larger value of the integration variable to a smaller one, as in the above integral with respect to $y$.

\section{Surface integrals}

The approach we have set up for line integrals can easily be extended to surface integrals. The key ingredient is to note that, for a given surface $S$, the vector surface element $d \overrightarrow{\boldsymbol{S}}$ is just the (appropriately ordered) cross product of the $d \overrightarrow{\boldsymbol{r}}$ vectors computed for (any!) two non-collinear families of curves lying in the surface. That is, $d \overrightarrow{\boldsymbol{S}}=\hat{\boldsymbol{n}} d S=d \overrightarrow{\boldsymbol{r}}_{1} \times d \overrightarrow{\boldsymbol{r}}_{2}$, where $d S$ is the (scalar) surface element of $S$, and $\hat{\boldsymbol{n}}$ is the unit normal vector to $S$ (with given orientation). While any families of curves will work, in practice it is important to choose ones that make it as easy as possible to express the limits of integration.

We first illustrate this approach on a problem typical of those in calculus textbooks. Consider finding the flux of the vector field $\overrightarrow{\boldsymbol{F}}=z \hat{\boldsymbol{k}}$ up through the part of the plane $x+y+z=1$ lying in the first octant. We begin with the infinitesimal vector displacement in rectangular coordinates in three dimensions, namely

$$
d \overrightarrow{\boldsymbol{r}}=d x \hat{\boldsymbol{\imath}}+d y \hat{\boldsymbol{j}}+d z \hat{\boldsymbol{k}}
$$

A natural choice of curves in this surface is given by setting $y$ or $x$ constant, so that $d y=0$ or $d x=0$, respectively. We thus obtain

$$
\begin{aligned}
& d \overrightarrow{\boldsymbol{r}}_{1}=d x \hat{\boldsymbol{i}}+d z \hat{\boldsymbol{k}}=(\hat{\boldsymbol{\imath}}-\hat{\boldsymbol{k}}) d x, \\
& d \overrightarrow{\boldsymbol{r}}_{2}=d y \hat{\boldsymbol{j}}+d z \hat{\boldsymbol{k}}=(\hat{\boldsymbol{j}}-\hat{\boldsymbol{k}}) d y,
\end{aligned}
$$

where we have used what we know (the equation of the plane) to determine each expression in terms of a single parameter. The surface element is thus

$$
d \overrightarrow{\boldsymbol{S}}=d \overrightarrow{\boldsymbol{r}}_{1} \times d \overrightarrow{\boldsymbol{r}}_{2}=(\hat{\boldsymbol{\imath}}+\hat{\boldsymbol{\jmath}}+\hat{\boldsymbol{k}}) d x d y
$$

and the flux becomes

$$
\iint_{S} \overrightarrow{\boldsymbol{F}} \cdot d \overrightarrow{\boldsymbol{S}}=\iint_{S} z d x d y=\int_{0}^{1} \int_{0}^{1-y}(1-x-y) d x d y=\frac{1}{6} .
$$

The limits were chosen by visualizing a projection of the surface into the $x y$-plane, which is a triangle bounded by the $x$-axis, the $y$-axis, and the line whose equation is $x+y=1$. Note that this latter equation is obtained from the equation of the surface by using what we know-namely that $z=0$.

Just as for line integrals, there is a rule of thumb that tells you when to stop using what you know to compute surface integrals: Don't start integrating until the integral is expressed in terms of two parameters, and the limits in terms of those parameters have been determined. Surfaces are two-dimensional! 


\section{Highly symmetric surfaces}

One of the most fundamental examples in electromagnetism is the electric field of a point charge $q$ at the origin, which is given by

$$
\overrightarrow{\boldsymbol{E}}=\frac{q}{4 \pi \epsilon_{0}} \frac{\hat{\boldsymbol{r}}}{r^{2}}=\frac{q}{4 \pi \epsilon_{0}} \frac{x \hat{\boldsymbol{\imath}}+y \hat{\boldsymbol{j}}+z \hat{\boldsymbol{k}}}{\left(x^{2}+y^{2}+z^{2}\right)^{3 / 2}},
$$

where $\hat{\boldsymbol{r}}$ is now the unit vector in the radial direction in spherical coordinates. Note that the first expression clearly indicates both the spherical symmetry of $\overrightarrow{\boldsymbol{E}}$ and its $1 / r^{2}$ falloff behavior, while the second expression does neither. Given the electric field, Gauss's Law allows one to determine the total charge inside any closed surface, namely

$$
\frac{q}{\epsilon_{0}}=\iint_{S} \overrightarrow{\boldsymbol{E}} \cdot d \overrightarrow{\boldsymbol{S}}
$$

which is of course just the Divergence Theorem.

As noted in the introduction, it is easy to determine $d \overrightarrow{\boldsymbol{S}}$ on the sphere by inspection; we nevertheless go through the details of the differential approach for this case. We use "physicists" conventions" for spherical coordinates, so that $\theta$ is the angle from the North Pole, and $\phi$ the angle in the $x y$-plane. We use the obvious families of curves, namely the lines of latitude and longitude. Starting either from the general formula for $d \overrightarrow{\boldsymbol{r}}$ in spherical coordinates

$$
d \overrightarrow{\boldsymbol{r}}=d r \hat{\boldsymbol{r}}+r \sin \theta d \theta \hat{\boldsymbol{\theta}}+r d \phi \hat{\boldsymbol{\phi}}
$$

or directly using the geometry behind that formula, one quickly arrives at

$$
d \overrightarrow{\boldsymbol{S}}=d \overrightarrow{\boldsymbol{r}}_{1} \times d \overrightarrow{\boldsymbol{r}}_{2}=r \sin \theta d \theta \hat{\boldsymbol{\theta}} \times r d \phi \hat{\boldsymbol{\phi}},
$$

so that

$$
\iint_{S} \overrightarrow{\boldsymbol{E}} \cdot d \overrightarrow{\boldsymbol{S}}=\int_{0}^{2 \pi} \int_{0}^{\pi} \frac{q}{4 \pi \epsilon_{0}} \frac{\hat{\boldsymbol{r}}}{r^{2}} \cdot r^{2} \sin \theta d \theta d \phi \hat{\boldsymbol{r}}=\frac{q}{\epsilon_{0}}
$$

Finally, we note that this approach can be extended to volume integrals using the triple product. The volume element becomes

$$
d V=\left(d \overrightarrow{\boldsymbol{r}}_{1} \times d \overrightarrow{\boldsymbol{r}}_{2}\right) \cdot d \overrightarrow{\boldsymbol{r}}_{3}
$$

for the $d \overrightarrow{\boldsymbol{r}}$ vectors computed for (any!) three non-coplanar families of curves.

\section{Less symmetric surfaces}

The reader may have the feeling that two quite different languages are being spoken here. The tilted plane was treated in essentially the traditional manner found in calculus textbooks, using rectangular coordinates. While the "use what you know" strategy may be somewhat unfamiliar, the basic idea should not be. On the other hand, the example in the previous section will be quite unfamiliar to most mathematicians, because of their use of adapted basis vectors such as $\hat{\boldsymbol{r}}$. Mathematicians should realize that mastering these examples helps students learn to look for symmetry and to argue 
geometrically. At the same time, mathematicians may not be satisfied by an approach that seems to be so intimately dependent on the particular problem being studied.

We argue, however, that the approach being presented here is much more flexible than may appear at first sight. We demonstrate this flexibility by integrating over a paraboloid, the classic example found in calculus textbooks.

We compute the flux of the axially symmetric vector field

$$
\overrightarrow{\boldsymbol{F}}=r \hat{\boldsymbol{r}}=x \hat{\boldsymbol{\imath}}+y \hat{\boldsymbol{J}}
$$

outwards through the part of the paraboloid $z=r^{2}$ lying below the plane $z=4$. The first thing we need is the formula for $d \overrightarrow{\boldsymbol{r}}$ in cylindrical coordinates, which is a straightforward generalization of (3) in polar coordinates, namely

$$
d \overrightarrow{\boldsymbol{r}}=d r \hat{\boldsymbol{r}}+r d \phi \hat{\boldsymbol{\phi}}+d z \hat{z}
$$

Next, we need two families of curves on the paraboloid; the natural choices are those with $r=$ constant or $\phi=$ constant, respectively. If $r$ is constant, so is $z$, and we have simply

$$
d \overrightarrow{\boldsymbol{r}}_{1}=r d \phi \hat{\phi}
$$

If $\phi$ is constant, there will be no $\hat{\phi}$ term in $d \vec{r}$, but we may still use what we know to compute $d z=2 r d r$, thus obtaining

$$
d \overrightarrow{\boldsymbol{r}}_{2}=d r \hat{\boldsymbol{r}}+2 r d r \hat{z} .
$$

Taking the cross product leads to

$$
d \overrightarrow{\boldsymbol{S}}=d \overrightarrow{\boldsymbol{r}}_{1} \times d \overrightarrow{\boldsymbol{r}}_{2}=\left(2 r^{2} \hat{\boldsymbol{r}}-r \hat{z}\right) d r d \phi,
$$

and at this point we must check that we have chosen the correct orientation. (We have, since the coefficient of $\hat{z}$ is negative.) The rest is easy: compute the dot product, determine the limits, and do the integral. This results in

$$
\iint_{S} \overrightarrow{\boldsymbol{F}} \cdot d \overrightarrow{\boldsymbol{S}}=\int_{0}^{2 \pi} \int_{0}^{2} 2 r^{3} d r d \phi=16 \pi
$$

It is of course also possible to use rectangular coordinates to determine $d \overrightarrow{\boldsymbol{S}}$, choosing curves on the paraboloid $z=x^{2}+y^{2}$ with $x=$ constant or $y=$ constant. The resulting integral cries out for polar coordinates-which turns it into the same integral as the above.

\section{Discussion}

The approach presented here emphasizes the geometry behind line and surface integration. Because the integration elements $d \overrightarrow{\boldsymbol{r}}$ and $d \overrightarrow{\boldsymbol{S}}$ are treated as geometric objects, it is easy to "use what you know" when dealing with the highly symmetric situations commonly encountered by physicists and engineers, especially at the undergraduate level. Nevertheless, this approach is flexible enough to handle less symmetric situations, such as the paraboloid. 
We have chosen to treat the vector differential $d \overrightarrow{\boldsymbol{r}}$ as fundamental without providing a rigorous definition to the student. This is quite similar to the treatment of substitution in single integrals, where differentials are often used as a mnemonic devicesomething that works, but isn't explicitly defined. We believe the combination of geometric intuition and flexibility provided by our approach is well worth the price.

Acknowledgments. This work was supported by NSF grants DUE-9653250 \& DUE0088901 and by the Oregon Collaborative for Excellence in the Preparation of Teachers (OCEPT). We especially thank OCEPT for organizing a writing workshop at which we wrote the initial draft, and Gowri Meda for helpful comments.

Our work has been greatly influenced by the formative comments of the National Advisory Committee to the Vector Calculus Bridge Project: David Griffiths, Harriet Pollatsek, and James Stewart. We have also benefitted from discussions with mathematics and physics faculty at Mount Holyoke College, and support from the Mount Holyoke College Hutchcroft Fund.

\section{References}

1. Tevian Dray and Corinne A. Manogue, The vector calculus gap, PRIMUS 9 (1999) 21-28.

2. James Stewart, Calculus, 4th ed., Brooks-Cole, 1999.

3. David J. Griffiths, Introduction to Electrodynamics, 3rd ed., Prentice-Hall, 1999.

4. H. M. Schey, Div, Grad, Curl and All That, 3rd ed., Norton, 1997.

5. William McCallum, Deborah Hughes-Hallett, Andrew Gleason, et al., Multivariable Calculus, 3rd ed. Wiley, 2001.

6. Tevian Dray and Corinne A. Manogue, Spherical coordinates, College Math J. 34 (2003) 168-169.

\section{Cross-product Humor}

Q. What do you get when you cross a chicken with a turkey?

A. Chicken turkey $\sin \theta$.

Q. What do you get when you cross a chicken with a mountain climber?

A. You can't do that. A mountain climber is a scaler.

Don't blame the editor. These things go the rounds, and represent the best that mathematical humorists can do. 